(1)

CrossMark

\title{
Inflammation and intussusceptive angiogenesis in COVID-19: everything in and out of flow
}

\author{
Maximilian Ackermann $\mathbb{1}^{1,2}$, Steven J. Mentzer ${ }^{3}$, Martin Kolb $\mathbb{1}^{4}$ and \\ Danny Jonigk ${ }^{5,6}$
}

Affiliations: ${ }^{1}$ Institute of Pathology and Molecular Pathology, Helios University Clinic Wuppertal, University of Witten/Herdecke, Wuppertal, Germany. ${ }^{2}$ Institute of Functional and Clinical Anatomy, University Medical Center of the Johannes Gutenberg-University Mainz, Mainz, Germany. ${ }^{3}$ Laboratory of Adaptive and Regenerative Biology, Harvard Medical School, Brigham and Women's Hospital, Boston, MA, USA. "Firestone Institute for Respiratory Health, Research Institute at St Joseph's Healthcare, Dept of Medicine, McMaster University, Hamilton, ON, Canada. ${ }^{5}$ Institute of Pathology, Hannover Medical School, Hannover, Germany. ${ }^{6}$ Member of the German Center for Lung Research (DZL), Biomedical Research in Endstage and Obstructive Lung Disease Hannover (BREATH), Hannover, Germany.

Correspondence: Maximilian Ackermann, University Medical Center of the Johannes Gutenberg-University Mainz, Institute of Functional and Clinical Anatomy, Johann-Joachim-Becher-Weg 13, 55128 Mainz; Institute of Pathology and Molecular Pathology, Helios University Clinic Wuppertal, University of Witten/Herdecke, Wuppertal, Germany. E-mail: maximilian.ackermannduni-mainz.de

@ERSpublications

Inflammation and intussusceptive angiogenesis in COVID-19 https://bit.ly/30lLh8K

Cite this article as: Ackermann $\mathrm{M}$, Mentzer SJ, Kolb $\mathrm{M}$, et al. Inflammation and intussusceptive angiogenesis in COVID-19: everything in and out of flow. Eur Respir J 2020; 56: 2003147 [https://doi.org/ $10.1183 / 13993003.03147-2020]$.

The human body contains 60000 miles of blood vessels, including at least 19 billion capillaries, so that under physiological conditions cells are located no further than 100-200 $\mu \mathrm{m}$ from the nearest capillary. In those, endothelial cells and pericytes seem to play a pivotal role in coronavirus disease 2019 (COVID-19) by binding severe acute respiratory syndrome coronavirus 2 (SARS-CoV-2) to the angiotensin-converting enzyme 2 (ACE2) $[1,2]$. In the lung, the transmembrane ACE2 receptor is predominantly expressed in endothelial cells, perivascular pericytes and type 2 cells $[2,3]$.

We recently showed that SARS-CoV-2 infection leads to angiocentric inflammation in COVID-19-induced respiratory failure, with a greater number of ACE2-positive endothelial cells compared to uninfected controls or to post mortem lung tissue from patients who succumbed to influenza A-related acute respiratory distress syndrome (ARDS) [1]. Although the detection of SARS-CoV-2 in post mortem tissue by transmission electron microscopy is a challenging task [4], replicated virus-like particles were observed enveloped in endothelial cells [1,5] and lymphatic cells [6], and also in type 2 and 1 pneumocytes [6, 7]. Increasing clinical evidence shows that endothelial dysfunction is a common denominator after SARS-CoV-2 infection in the multi-organ complexity and severity of COVID-19 [3]. COVID-19-related endothelial dysfunction is characterised by acute vascular inflammation and perivascular T-cell recruitment leading to disruption of the alveolar-capillary barrier and increased permeability [1-3]. The endothelial cells surrounded by T-lymphocytes show features of strong activation referred to as "endothelialitis", a process typically seen during rejection of solid organ transplants. The infection of

Copyright $\odot$ ERS 2020. This article is open access and distributed under the terms of the Creative Commons Attribution Non-Commercial Licence 4.0. 
endothelial cells by SARS-CoV-2 results in swelling and disruption of the endothelial cell barriers, an anomalous microvascular architecture, and an endothelial dysfunction $[1,3]$. These vascular injuries are accompanied by thrombosis, vasoconstriction and distinct intussusceptive angiogenesis, a unique rapid process of blood vessel neoformation by splitting a vessel in two lumens by an incorporation of circulating angiogenic cells (figure 1a) $[1,3]$. In the pulmonary vasculature from autopsies of COVID-19 patients, we observed a distinct occurrence of intussusceptive angiogenesis not only in early SARS-CoV-2 infected lungs, but also in lung tissue with an infection lasting more than 20 days. Beyond these findings in COVID-19 post mortem lung tissue, we revealed distinctive features of compensatory angiogenesis by intussusception in many other organs such as heart, liver, kidney, brain and lymphoreticular organs in patients who succumbed to COVID-19. The chaotic vessel regulation of focally vasoconstricted and progressively dilated vessel segments results in severe disturbances of physiological laminar flow. Two major forms of thrombi have been reported so far in COVID-19 patients [8]. Pulmonary embolism in larger pulmonary vessels probably based on deep vein thrombosis were seen in a minority of COVID-19 patients, whereas the vast majority demonstrated platelet aggregates obstructing the microvasculature and peripheral vascularity caused by fibrin strands, activated platelets, deformed neutrophils and neutrophil extracellular traps $[8,9]$. Viral-associated thrombotic microangiopathies have been described in numerous inflammatory cardiorespiratory diseases (e.g. influenza [10] or myocarditis [11]). We compared post mortem lung tissues from patients who died from COVID-19 with severe ARDS and diffuse alveolar damage due to influenza A (H1N1) infection. Thereby, we found nine times more microthrombi in COVID-19 lungs compared to influenza A (H1N1) lungs [1]. The microangiopathy observed in COVID-19 patients, specifically, the vasoconstriction and clotting in smaller blood vessels, results in hypoxia, shunting and an increase of pulmonary vascular resistance [8]. Interestingly, COVID-19 does not show characteristics of a "typical" ARDS [12]. The discrepancy between a general well-preserved lung mechanics and the severity of hypoxaemia could be explained by a decreased capacity of vascular tone in venules and the capillary plexus. Our molecular data on COVID-19 lung tissue gave evidence of a significant upregulation of vasoconstrictive mediators such as prostaglandins (phospholipase A,
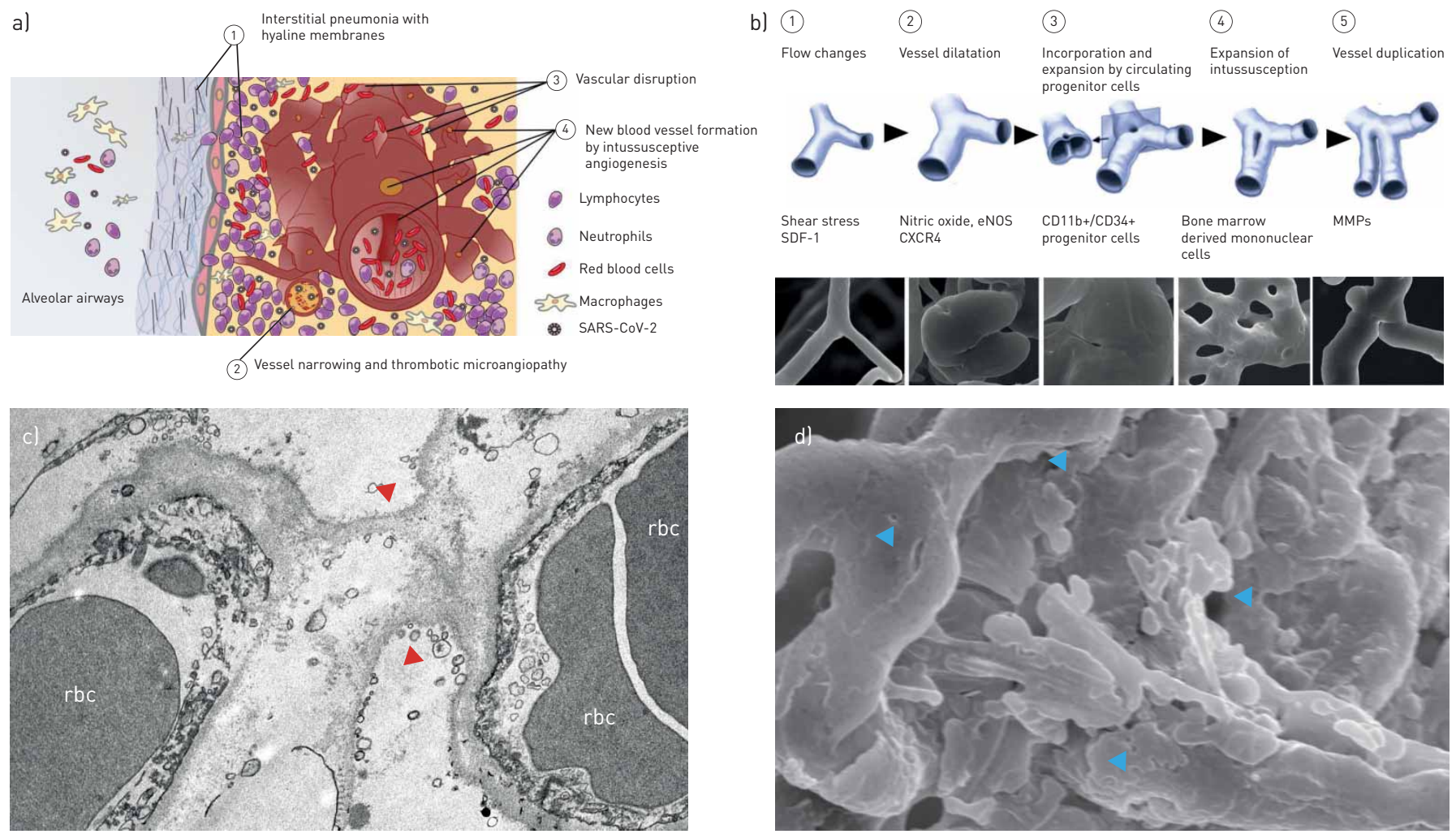

FIGURE 1 a) Schematic of pulmonary endothelialitis, thrombosis, and intussusceptive angiogenesis in coronavirus disease 2019 (COVID-19). SARS-CoV-2: severe acute respiratory syndrome coronavirus 2. b) Intussusceptive angiogenesis is a morphogenetic process which rapidly expands the vascular plexus. c) Transmission electron micrograph of lung tissue of a deceased COVID-19 patient highlights the formation of an intussusceptive pillar (red arrowheads) which spans the lumen of the vascular walls. rbc: red blood cells. d) A disrupted vascularity with distorted vessels and intussusceptive pillars (blue arrowheads) is observed in COVID-19 lungs, as depicted as scanning electron micrograph of microvascular corrosion casts of COVID-19 autopsies. 
leukotrienes) [1], as well as an increase of nitric oxide synthase (NOS). Nitric oxide is produced in endothelial cells by the enzyme endothelial nitric oxide synthase (eNOS) or in monocytes and macrophages by inducible nitric oxide synthase (iNOS). The rheologic properties of blood flow (laminar versus turbulent) and vessel morphology determine the shear stress on the vascular wall [13]. In general, high shear stress, as observed in physiological laminar flow, is considered angioprotective, promoting endothelial cell survival, vasodilation and anticoagulation [14, 15]. Low shear stress, on the other hand, results in the secretion of vasoconstrictors, platelet aggregation, coagulation and pathological reshaping of microvascular architecture [15-17]. The pathological consequences of these blood flow dynamics have been described in many diseases, such as atherosclerosis [18], inflammatory diseases [15, 17] and malignancies [16]. Our own haemodynamic studies on inflammatory-related changes of the blood flow $[13,14,17]$ revealed heterogeneity in flow patterns, with dispersed flow velocities, occluded vessel segments and platelet aggregates associated with upregulation of thrombotic agonists.

The structural adaption of the microvascular architecture, the transmigration of lymphocytes and the "cytokine storm" observed in COVID-19 patients is a response to SARS-CoV-2-induced cellular damage. Many cytokines appear to be involved in enhancing lymphocyte recruitment. Tumour necrosis factor $\alpha$ $(\mathrm{TNF} \alpha)$ is known to increase the adhesion of lymphocytes by activating the SDF-1/CXCR4 pathways. The T-lymphocyte/ endothelial interaction likely contributes to the prolonged interstitial inflammation in COVID-19. Activated T-cells attracted by chemotactic chemokines (e.g. CCL17, CCL8 or CCR1) [1] preferentially adhere to activated endothelial cells $[19,20]$. Despite an increase in inflammatory blood flow and increased wall shear stress, transendothelial lymphocyte recruitment can occur in selected capillary beds (liver and lung), post-capillary venules (most parenchymal organs), and even specialised vascular segments that acquire structural modifications that reduce flow velocity and wall shear stress [21]. Therefore, the structure of the microcirculation is continuously adapting to metabolic demands and immunosurveillance. The close association of inflammation and angiogenesis represents a pivotal pillar in perpetuating inflammatory processes during wound healing and infections such as COVID-19. Inflamed human endothelial cells and pericytes express high levels of toll-like receptors (TLRs) which are recognised together with their intracellular adaptor protein MyD88 as sentinels of the innate immune system [22]. SARS-CoV and other coronaviruses may be recognised by TLRs and MyD88 [23, 24]. Stimulation of endothelial TLRs and MyD88 results in a release of cytokines (e.g. interferon $\gamma$, TNF $\alpha$, interleukin $1 \alpha$, granulocyte colony-stimulating factor), chemokines, leukocyte adhesion molecules (e.g. E-selectin, intercellular adhesion molecule 1, vascular cell adhesion protein 1), procoagulation mediators (e.g. fibrin, plasminogen activator inhibitor, von Willebrand factor), and proangiogenic factors (e.g. vascular endothelial growth factor (VEGF), NOS or CD14 monocytes) [25].

"Intussusceptive" (non-sprouting) angiogenesis is a well-characterised morphogenetic process in cancer [26], inflammatory diseases and tissue regeneration [27]. Distinct from intussusceptive angiogenesis, sprouting angiogenesis is characterised by sprouts composed of endothelial cells. The endothelial sprouts typically grow toward an angiogenic stimulus (such as VEGF-A) and add vessels to tissues devoid of blood vessels. Intussusceptive angiogenesis is a rapid process of intravascular septation that produces two lumens from a single vessel within minutes. The process appears to recruit bone-marrow derived mononuclear cells, expanding and adapting capillary plexuses without requiring active proliferation of endothelial cells (figure 1b) [28]. The newly formed "intussusceptive pillars" (figure 1c) are then permeated by pericytes and myofibroblasts providing mechanical stabilisation of the transcapillary pillar core. We previously showed that this formation of intussusceptive pillars is primarily located in dilated vascular segments with low blood flow velocity and reduced wall shear stress $[12,13]$. Recently, CXCL12/CXCR4 signalling has been identified as an important molecular regulator of intussusceptive angiogenesis and hypoxia [29]: the positive feedback loop between vascular shear stress, CXCL12 (SDF1) expression, hypoxia and the release of eNOS has been identified as an adaptation of the vascular system to maintain blood flow responsive to the demands of prolonged inflammation. Therefore, the pronounced release of eNOS cascade is a pivotal physiological process to maintain blood flow into tissues with occluded vessels and to initiate tissue repair by expanding the vascular architecture by intussusceptive angiogenesis. In our own studies, we observed abundant intussusceptive angiogenesis in the disrupted pulmonary vascular architecture of patients who died of COVID-19 (figure $1 \mathrm{c}$ and d), stated in numbers nearly three times higher than in influenza A (H1N1) lungs. Furthermore, the expression of CXCL12 and CXCR4 was highly upregulated in these COVID-19 lungs and was associated with dense T-cell infiltration. These findings are consistent with inflammation-induced angiogenesis observed in other conditions, such as colitis $[30,31]$ and malignant tumours [26].

In a recent morphomolecular study published in the European Respiratory Journal [32], we demonstrated the presence and impact of microvascular alterations in fibrotic interstitial lung diseases. We observed a higher frequency of intussusceptive features in the injury patterns of nonspecific interstitial pneumonia 


\begin{tabular}{lccccc}
\multicolumn{1}{l}{ TABLE 1 Histological features of lung diseases relevant to intussusceptive angiogenesis } \\
& $\begin{array}{l}\text { Fatal } \\
\text { hypoxia }\end{array}$ & Lymphocytes & Endothelialitis & $\begin{array}{c}\text { Microthrombi } \\
\text { Intussusceptive } \\
\text { angiogenesis }\end{array}$ \\
\hline Influenza A [1] & Yes & Yes & & \\
NSIP [32] & Yes & Yes & & & $\leftrightarrow$ \\
AFE [32] & Yes & Yes & & Yes & $\uparrow \uparrow$ \\
UIP [32] & Yes & & Yes & $\uparrow$ \\
CTEPH [34] & Yes & & Yes & Yes & $\uparrow$ \\
PCH [35] & Yes & & Yes & Yes & $\uparrow$ \\
PVOD [35] & Yes & & Yes & Yes & $\uparrow \uparrow \uparrow$ \\
COVID-19 [1] & Yes & Yes & Yes
\end{tabular}

NSIP: nonspecific interstitial pneumonia; AFE: alveolar fibroelastosis; UIP: usual interstitial pneumonia; CTEPH: chronic thromboembolic pulmonary hypertension; $\mathrm{PCH}$ : pulmonary capillary haemangiomatosis; PVOD: pulmonary veno-occlusive disease; COVID-19: coronavirus disease 2019.

and alveolar fibroelastosis fibrotic lungs, whereas usual interstitial pneumonia lungs revealed compensatory angiogenesis predominantly by sprout formation [32, 33]. In addition, intussusceptive angiogenesis was observed in chronic pulmonary vascular diseases with variable degree of thrombosis, such as chronic thromboembolic pulmonary hypertension [34], pulmonary capillary haemangiomatosis [35, 36], and pulmonary veno-occlusive disease (table 1) [35]. Although the pathological mechanisms underlying fibrotic remodelling in pulmonary thromboembolic occlusions are poorly understood, thrombofibrosis and endothelial-mesenchymal transition seem to be promoted by hypoxia-induced activation of endothelial cells, intussusceptive angiogenesis, activation of mesenchymal cells and immune cells [31-34]. There is a compelling evidence that at least the progress and severity of progressive interstitial lung disease may be influenced by coagulation and fibrinolytic capacities and vascular permeability [37-39], although the therapeutic use of orally administered anticoagulants has been critically evaluated in idiopathic pulmonary fibrosis patients [40]. Especially in the light of inestimable long-term complications in COVID-19, further experimental and observational studies should investigate the contribution and the interplay between the overwhelming angiocentric T-cell inflammation, thrombotic microangiopathy and the compensatory flow-regulated intussusceptive angiogenesis in the increased morbidity and mortality of COVID-19.

Conflict of interest: M. Ackermann has nothing to disclose. S.J. Mentzer has nothing to disclose. M. Kolb reports grants and personal fees from Roche, Boehringer Ingelheim and Prometic, grants from GSK, Respivert, Alkermes, Pharmaxis and Canadian Institute for Health Research, personal fees from Genoa, Indalo, Third Pole and Pieris, outside the submitted work. D. Jonigk has nothing to disclose.

\section{References}

1 Ackermann M, Verleden SE, Kuehnel M, et al. Pulmonary vascular endothelialitis, thrombosis, and angiogenesis in Covid-19. N Engl J Med 2020; 383: 120-128.

2 Cardot-Leccia N, Hubiche T, Dellamonica J, et al. Pericyte alteration sheds light on micro-vasculopathy in COVID-19 infection. Intensive Care Med 2020; 46: 1777-1778.

3 Huertas A, Montani D, Savale L, et al. Endothelial cell dysfunction: a major player in SARS-CoV-2 infection (COVID-19)? Eur Respir J 2020; 56: 2001634.

4 Goldsmith CS, Miller SE, Martines RB, et al. Electron microscopy of SARS-CoV-2: a challenging task. Lancet 2020; 395: e99.

5 Ackermann M, Mentzer SJ, Jonigk D. Pulmonary vascular pathology in Covid-19. Reply. N Engl J Med 2020; 383: 888-889.

6 Martines RB, Ritter JM, Matkovic E, et al. Pathology and pathogenesis of SARS-CoV-2 associated with fatal coronavirus disease, United States. Emerging Infect Dis 2020; 26: 2005-2015.

7 Bradley BT, Maioli H, Johnston R, et al. Histopathology and ultrastructural findings of fatal COVID-19 infections in Washington State: a case series. Lancet 2020; 396: 320-332.

8 Price LC, McCabe C, Garfield B, et al. Thrombosis and COVID-19 pneumonia: the clot thickens! Eur Respir J 2020; 56: 2001608.

9 Leppkes M, Knopf J, Naschberger E, et al. Vascular occlusion by neutrophil extracellular traps in COVID-19. EBioMedicine 2020; 58: 102925.

10 Sugiyama MG, Gamage A, Zyla R, et al. Influenza virus infection induces platelet-endothelial adhesion which contributes to lung injury. J Virol 2016; 90: 1812-1823.

11 Ackermann M, Wagner WL, Rellecke P, et al. Parvovirus B19-induced angiogenesis in fulminant myocarditis. Eur Heart J 2020; 41: 1309.

12 Gattinoni L, Coppola S, Cressoni M, et al. COVID19 does not lead to a typical acute respiratory distress syndrome. Am J Respir Crit Care Med 2020; 201: 1299-1300. 
13 Lee GS, Filipovic N, Miele LF, et al. Blood flow shapes intravascular pillar geometry in the chick chorioallantoic membrane. J Angiogenes Res 2010; 2: 11.

14 Filipovic N, Tsuda A, Lee GS, et al. Computational flow dynamics in a geometric model of intussusceptive angiogenesis. Microvasc Res 2009; 78: 286-293.

15 Ravnic DJ, Konerding MA, Tsuda A, et al. Structural adaptations in the murine colon microcirculation associated with hapten-induced inflammation. Gut 2007; 56: 518-523.

16 Jain RK, Tong RT, Munn LL. Effect of vascular normalization by antiangiogenic therapy on interstitial hypertension, peritumor edema, and lymphatic metastasis: insights from a mathematical model. Cancer Res 2007; 67: 2729-2735.

17 Miele LF, Turhan A, Lee GS, et al. Blood flow patterns spatially associated with platelet aggregates in murine colitis. Anat Rec (Hoboken) 2009; 292: 1143-1153.

18 Napoli C, Ignarro LJ. Nitric oxide and pathogenic mechanisms involved in the development of vascular diseases. Arch Pharm Res 2009; 32: 1103-1108.

19 von Andrian UH, Mackay CR. T-cell function and migration. Two sides of the same coin. N Engl J Med 2000; 343: 1020-1034.

20 Li X, Abdi K, Rawn J, et al. LFA-1 and L-selectin regulation of recirculating lymphocyte tethering and rolling on lung microvascular endothelium. Am J Respir Cell Mol Biol 1996; 14: 398-406.

21 Secomb TW, Konerding MA, West CA, et al. Microangiectasias: structural regulators of lymphocyte transmigration. Proc Natl Acad Sci USA 2003; 100: 7231-7234.

22 Ranchoux B, Antigny F, Rucker-Martin C, et al. Endothelial-to-mesenchymal transition in pulmonary hypertension. Circulation 2015; 131: 1006-1018.

23 Andonegui G, Bonder CS, Green F, et al. Endothelium-derived Toll-like receptor-4 is the key molecule in LPS-induced neutrophil sequestration into lungs. J Clin Invest 2003; 111: 1011-1020.

24 Butchi N, Kapil P, Puntambekar S, et al. Myd88 initiates early innate immune responses and promotes CD4 T cells during coronavirus encephalomyelitis. J Virol 2015; 89: 9299-9312.

25 Khakpour S, Wilhelmsen K, Hellman J. Vascular endothelial cell Toll-like receptor pathways in sepsis. Innate Immun 2015; 21: 827-846.

26 Ackermann M, Morse BA, Delventhal V, et al. Anti-VEGFR2 and anti-IGF-1R-Adnectins inhibit Ewing's sarcoma A673-xenograft growth and normalize tumor vascular architecture. Angiogenesis 2012; 15: 685-695.

27 Ackermann M, Houdek JP, Gibney BC, et al. Sprouting and intussusceptive angiogenesis in postpneumonectomy lung growth: mechanisms of alveolar neovascularization. Angiogenesis 2014; 17: 541-551.

28 Mentzer SJ, Konerding MA. Intussusceptive angiogenesis: expansion and remodeling of microvascular networks. Angiogenesis 2014; 17: 499-509.

29 Dimova I, Karthik S, Makanya A, et al. SDF-1/CXCR4 signalling is involved in blood vessel growth and remodelling by intussusception. J Cell Mol Med 2019; 23: 3916-3926.

30 Konerding MA, Turhan A, Ravnic DJ, et al. Inflammation-induced intussusceptive angiogenesis in murine colitis. Anat Rec (Hoboken) 2010; 293: 849-857.

31 Ackermann M, Tsuda A, Secomb TW, et al. Intussusceptive remodeling of vascular branch angles in chemically-induced murine colitis. Microvasc Res 2013; 87: 75-82.

32 Ackermann M, Stark H, Neubert L, et al. Morphomolecular motifs of pulmonary neoangiogenesis in interstitial lung diseases. Eur Respir J 2020; 55: 1900933.

33 Yanagihara T, Jones KD. Demystifying morphomolecular alterations of vasculature in interstitial lung diseases. Eur Respir J 2020; 55: 1902446.

34 Ackermann M, Gaumann A, Mentzer SJ, et al. Plexiform vasculopathy in chronic thromboembolic pulmonary hypertension. Am J Respir Crit Care Med 2017; 196: e48-e51.

35 Neubert L, Borchert P, Shin HO, et al. Comprehensive three-dimensional morphology of neoangiogenesis in pulmonary veno-occlusive disease and pulmonary capillary hemangiomatosis. J Pathol Clin Res 2019; 5: 108-114.

36 Weatherald J, Dorfmüller P, Perros F, et al. Pulmonary capillary haemangiomatosis: a distinct entity? Eur Respir Rev 2020; 29: 190168.

37 Bochenek ML, Rosinus NS, Lankeit M, et al. From thrombosis to fibrosis in chronic thromboembolic pulmonary hypertension. Thromb Haemost 2017; 117: 769-783.

38 Wuyts WA, Agostini C, Antoniou KM, et al. The pathogenesis of pulmonary fibrosis: a moving target. Eur Respir J 2013; 41: 1207-1218

39 Probst CK, Montesi SB, Medoff BD, et al. Vascular permeability in the fibrotic lung. Eur Respir J 2020; 56: 1900100.

40 Kreuter M, Wijsenbeek MS, Vasakova M, et al. Unfavourable effects of medically indicated oral anticoagulants on survival in idiopathic pulmonary fibrosis. Eur Respir J 2016; 47: 1776-1784. 